\title{
Synopsis of the genus Pycnolejeunea (Spruce) Schiffn. (Lejeuneaceae, Marchantiophyta) in Brazil
}

\author{
Cid José Passos Bastos ${ }^{1,3}$, (D) Adriele Lima Gentil1, (D) Adriel M. Sierra ${ }^{2}$ e (D) Charles E. Zartman²
}

Received: 28.12.2019; accepted: 10.07.2020

How to cite: Bastos, C.J.P., Gentil, A.L., Sierra, A.M. \& Zartman, C.E. 2020. Synopsis of the genus Pycnolejeunea (Spruce) Schiffn. (Lejeuneaceae, Marchantiophyta) in Brazil. Hoehnea 47: e1132019. http://dx.doi.org/10.1590/2236-8906-113/2019

\begin{abstract}
Synopsis of the genus Pycnolejeunea (Spruce) Schiffn. (Lejeuneaceae, Marchantiophyta) in Brazil). Pycnolejeunea is a pantropical genus represented in Brazil by nine species, according to the present treatment. Pycnolejeunea chocoensis M.E. Reiner \& Gradst., recently described for Colombia, is being cited for the first time for Brazil. Descriptions are given for all species and illustrations only for Pycnolejeunea chocoensis, Pycnolejeunea contigua (Nees) Grolle, Pycnolejeunea papillosa X.-L. He and Pycnolejeunea porrectilobula C.J. Bastos \& O. Yano. A key to the species of Pycnolejeunea occurring in Brazil is also presented.
\end{abstract}

Keywords: liverworts, neotropic, Porellales, taxonomy

RESUMO - (Sinopse do gênero Pycnolejeunea (Spruce) Schiffn. (Lejeuneaceae, Marchantiophyta). Pycnolejeunea é um gênero pantropical e está representado no Brasil por nove espécies, de acordo com o presente tratamento. Pycnolejeunea chocoensis M.E. Reiner \& Gradst., descrita recentemente para Colômbia, está sendo citada pela primeira vez para o Brasil. Descrições são apresentadas para todas as espécies e ilustração apenas para Pycnolejeunea contigua (Nees) Grolle, Pycnolejeunea chocoensis, Pyconolejeunea papillosa X.-L. He e Pycnolejeunea porrectilobula C.J. Bastos \& O. Yano. Uma chave para identificação das espécies de Pycnolejeunea que ocorrem no Brasil é também apresentada.

Palavras-chave: hepáticas, neotrópico, Porellales, taxonomia

\section{Introduction}

Pycnolejeunea (Spruce) Schiffn. (Lejeuneaceae) is a pantropical genus of 14 accepted species (Söderström et al. 2016, Bastos \& Zartman 2017). The genus was monographed by He (1999) who recognized nine species: five restricted to the Neotropics, three in Asia and Australia, and P. contigua (Nees) Grolle, with a wide, pantropical distribution. However, recently two new species were described: P. remotistipula C.J. Bastos \& Zartman (Bastos \& Zartman, 2017), from the Amazon in Brazil and Pycnolejeunea chocoensis M.E. Reiner \& Gradst. (Reiner-Drehwald \& Gradstein 2018) from Chocó in Colombia. A molecular phylogenetic analysis supported the reinstatement of Cheilolejeunea decurviloba
(Steph.) X.-L. He as a member of Pycnolejeunea (Ye et al. 2015). To date, Brazil is known to host nine species (in the present treatment), four of which are presently considered to be restricted to the Amazon Region: P. gradsteinii (State of Pará), P. chocoensis, P. remotistipula, and P. decurviloba (State of Amazonas) (Bastos \& Zartman 2017).

Molecular phylogenetic analysis of Lejeuneaceae resolved Pycnolejeunea as monophyletic within the tribe Lejeuneeae (Schäfer-Verwimp et al. 2014). The genus is supported by the following morphological synapomorphies: (a) leaf lobe mostly convex; (b) leaf lobe cells mamillose or papillose, rarely plane, with distinct trigones; (c) ocelli present in leaf lobes and female bracts, basal or suprabasal, rarely scattered or absent; (d) first lobule tooth prominent,

1. Universidade Federal da Bahia, Instituto de Biologia, Laboratório de Taxonomia de Briófitas - BrioFLORA, Campus Universitário de Ondina, Avenida Adhemar de Barros, s/n, Ondina, 40170-280 Salvador, BA, Brasil

2. Instituto Nacional de Pesquisas da Amazônia, Departamento de Biodiversidade, Av. André Araújo, 2936, Aleixo, CEP 69060-001, Manaus, AM, Brasil

3. Corresponding author: cidbastos@gmail.com 
the second tooth embedded at lobule free margin and usually indistinct; (e) underleaves usually imbricate, rarely distant; (f) pycnolejeuneoid innovations; (g) perianths 5-keeled, keels smooth to slightly crenate.

In the context of the current studies on the Lejeuneaceae of Brazil, we have recently discovered several novelties for Pycnolejeunea. Here we present a taxonomic treatment of Pycnolejeunea species known from Brazil, including detailed morphological descriptions and illustrations. Herein we also report Pycnolejeunea chocoensis as a new record for Brazil.

\section{Material and methods}

This study is largely based on examination of type material and herbarium specimens from ALCB, F, G, INPA, MG, SP, and RB. Fresh material has also been examined from recent collections.

The preliminary species list of Pycnolejeunea in Brazilian Flora 2020 (Bastos 2019) served as a baseline for the study. For each species the following information is provided: descriptions, presently recognized synonyms, references to published illustrations, general comments, global and regional (Brazilian) distributions (based on collections studied and on literature), vegetation types, substrate types and other selected material. Distribution for Brazil is based on geopolitical regions following the IBGE (Northern, Northeastern, Middle-Western, Southeastern, Southern). Accepted names follows Söderström et al. $(2015,2016)$.

Selected species of Pycnolejeunea were observed and digitally photographed following sputter coating with gold, using scanning electron microscope (SEM) JEOL JSM$661 \mathrm{OLV}$, in the Laboratório Multiusuário de Microscopia Eletrônica (LAMUME), of the Instituto de Física da Universidade Federal da Bahia (Institute of Physics, Federal University of Bahia).

\section{Results and Discussion}

In the present study nine species of Pycnolejeunea are recognized for Brazil, representing nearly ca. $64,3 \%$ of the total species described in worldwide. The sub-generic and sectional classification presented by He (1999) is not followed in the present treatment, however, the species concept of Pycnolejeunea and their nomenclatural synonyms as defined by He (1999) are followed herein.

To date, $P$. chocoensis, P. gradsteinii, $P$. decurviloba, and $P$. remotistipula occur only in the Amazon region of Brazil. However, P. decurviloba has a broader distribution, also occurring in Central America, and P. chocoensis is disjunct from the Chocó region of Colombia, thus being the first reference of the species in Brazil.

Pycnolejeunea species commonly occur in ombrophylous forest, seasonal forest, cerrado and restinga, usually growing on live tree trunk, liana, decomposing logs, and rarely on leaves ( $P$. contigua and P. macroloba, according to Zartman \& Ilkiu-Borges 2007).
Some of the distinctive characters of Pycnolejeunea, such as large trigones, pycnolejeuneoid innovations and mamillose to papillose leaf lobe cells, are also found in Cheilolejeunea. They differ, however, in their lobular structure relative to the first and the second teeth and, consequently, in the relative position of the hyaline papilla. In the majority of members of Lejeuneaceae, specifically in the tribe Lejeuneeae, the free margin of the lobule bears one or two teeth, which are separated or rarely lightly associated (He 1996, Bastos 2010, Renner 2012). In some taxa, however, one or both lobule teeth can be reduced. Mizutani (1961) found that the hyaline papilla is always proximal to the first tooth, either marginal or ental (on the inner side of the lobule) and distal to the second tooth. In other words, the position of the hyaline papilla depends on whether the first or the second lobule tooth is prominent. In Pycnolejeunea the first tooth is prominent and the second tooth is reduced.

According to He (1999), the ocelli of Pycnolejeunea are generally variable in number and may occur isolated or aggregated basally (confined to the leaf lobe base) to suprabasally near leaf lobe base to the proximal half of the leaf lobes, and female bracts.

Although the presence of ocelli is an important and diagnostic feature of Pycnolejeunea, in some species $(P$. decurviloba, $P$. remotistipula, and $P$. chocoensis) they are always absent and others, such as $P$. contigua, occasionally absent.

According to the widely accepted concept, mammilla is a term restricted to hollow cell wall elevations without thickening, i.e., the cell lumen penetrates the cavity, whereas papillae are solid projections of the cell wall (Câmara \& Kellogg 2010). This concept is accepted in the present treatment.

\section{Taxonomic treatment}

Pycnolejeunea (Spruce) Schiffn. in Engler \& Prantl, Nat. Pflanzenfam. 1(3): 124. 1893.

$\equiv$ Lejeunea subg. Pycnolejeunea Spruce, Trans. \& Proc. Bot. Soc. Edinburgh 15: 246. 1884.

Plants small to robust, pale green to yellowish green, brown to dark brown when dry, pale green to yellowish green, brown to dark brown when dry, vegetative branches Lejeunea-type. Stem in cross section with 7-14(-27) epidermal cells and 9-28(-57) medullary cells, thickwalled; ventral merophyte 2(-4)-8 wide. Leaves imbricate to contiguous, rare distant, wide-spreading; leaf lobe suborbicular, ovate, oblong-ovate to falcate, dorsal and ventral margin entire to slightly crenulate, apex rounded, curved to plane; lobe cells mammillose to papillose (uni or pluripapillose), rarely plane, rounded to hexagonal, trigones small to large, intermediate thickenings present or absent; ocelli mostly large, ovate to oblong, (0-)1-16(-24), isolate to aggregated basal to suprabasal, rarely absent; oil bodies 1-3 per cell, fusiform, large, segmented; leaf lobule small 
to large, ovate to rectangular to conic, inflated, free margin plane to involute, tooth (first tooth) unicellular, mostly short, hyaline papilla marginal, keel arched to straight, smooth to crenulate. Underleaves imbricate to contiguous, small to large, ovate, orbicular to reniform, mostly wider than long, bifid, sinus acute, outer margins entire, insertion line arched. Androecia on short branches, terminal to intercalary,
3-11(-40) pars of bracts, bracteoles 1-2 restricted to the base of the branch. Gynoecia on short or long branches, 1-2 pycnolejeuneoid innovations, lobe bracts ovate, obovate to orbicular, ocelli present or absent, bracteole obovate to ovate, bifid; perianths obovate, 5-keeled, keels smooth, beak short. Vegetative reproduction by caducous leaves or regenerants.

Key to species of Pycnolejeunea from Brazil

\section{Leaf lobule decurved; ocelli absent, rarely present}

2. Ventral merophyte two cells wide; underleaves distant, widely ovate P. chocoensis

2. Ventral merophyte 4-6 cells wide; underleaves imbricate, reniform P. decurviloba

1. Leaf lobule conic, ovate, oblong, ovate, rectangular, rectangular-ovate, not decurved; ocelli present, rarely absent

3. Ventral merophyte $4-8$ cells wide P. densistipula

3. Ventral merophyte two cells wide

4. Leaf lobule rectangular or rectangular-ovate, $1 / 2-2 / 3$ of the leaf length, free margin composed by $9-20$ elongated cells 5. Underleaves small (170-210 $\mu \mathrm{m}$ wide), distant, underleaf lobes apex rounded; ocelli absent ..... P. remotistipula

5. Underleaves larger (320-360 $\mu \mathrm{m}$ wide), imbricate to contiguous, underleaf lobes apex acute; ocelli present ....

P. macroloba

4. Leaf lobule ovate to conic, up to $1 / 2$ of the leaf length, free margin composed by 4-8(-9) elongated or rectangular cells 6. Leaf lobule ovate

7. Leaf cells strongly papillose, with high papillae; underleaves imbricate, orbicular to reniform P. papillosa

7. Leaf cells weakly papillose, with low papillae; underleaves imbricate to contiguous, ovate to suborbicular P. contigua

6. Leaf lobule conic, elongate

8. Leaf cells unipapillose; leaf lobe suborbicular to ovate, not falcate, plane; underleaves distant to contiguous, cuneate base ..... . P. gradsteinii

8. Leaf cells pluripapillose; leaf lobe oblong-ovate, falcate, convex; underleaves imbricate to contiguous, rounded base P. porrectilobula

1. Pycnolejeunea chocoensis M.E.Reiner \& Gradst., Cryptogamie, Bryologie 39(3): 326. 2018. Type: COLOMBIA: Departamento Chocó, municipio Nuquí, around Biological Station "El Amargal", coast lowland rainforest, ca 30 m.s.s.m., on liana, 6-VIII-1992, S.R. Gradstein 8877 (holotype: COL, not seen).

Figure $1 \mathrm{a}, \mathrm{b}$

Description and illustration: Reiner-Drehwald \& Gradstein (2018).

Plants 1.0-1.3 mm wide, vegetative branches Lejeuneatype. Stem $100-110 \mu \mathrm{m}$ in diameter, in cross section with 7 epidermal cells and 11-12 medullary cells, thin-walled; ventral merophyte two cells wide. Leaves imbricate, widespreading; leaf lobe ovate to ovate-oblong, 550-750 $\mu \mathrm{m}$ long $\times 490-630 \mu \mathrm{m}$ wide, dorsal margin slightly arched, entire, ventral margin straight to slightly concave, entire, apex rounded; leaf cells rounded to oblong, 23-55 $\times 23-43 \mu \mathrm{m}$, thin-walled, plane, trigones large, 1-2 intermediate thickenings; ocelli mostly absent, rarely present, (0-)1-2 basal, 50-60 × $38 \mu \mathrm{m}$; oil bodies not seen; leaf lobule ovate, inflated, decurved, small, 135-140 $\mu \mathrm{m}$ long $\times 130-135 \mu \mathrm{m}$ wide, free margin plane, composed by 5-7 rectangular cells to subrectangular, tooth short, hyaline papilla large, oblong, keel straight; reduce lobules frequent. Underleaves distant, widely ovate, wider than long, $165-255 \mu \mathrm{m}$ long $\times 245$ $260 \mu \mathrm{m}$ wide, bifid to $1 / 2$ of its length, sinus acute, bases rounded, insertion line curved. Androecia and gynoecia not seen. Leaves with marginal rhizoids frequent.

Pycnolejeunea chocoensis can be recognized by the decurved leaf lobule (the lobule apex is positioned towards the ventral margin of the leaf lobe), absence of ocelli (in some specimens examined from Amazon, A.M. Sierra 5104, C.E. Zartman 10232, 1-2 ocelli were observed) and ventral merophytes with two cells wide. $P$. chocoensis is similar to $P$. decurviloba (both species has decurved leaf lobules and lack of ocelli), but the latter species is a larger plant with reniform underleaves and ventral merophyte with 

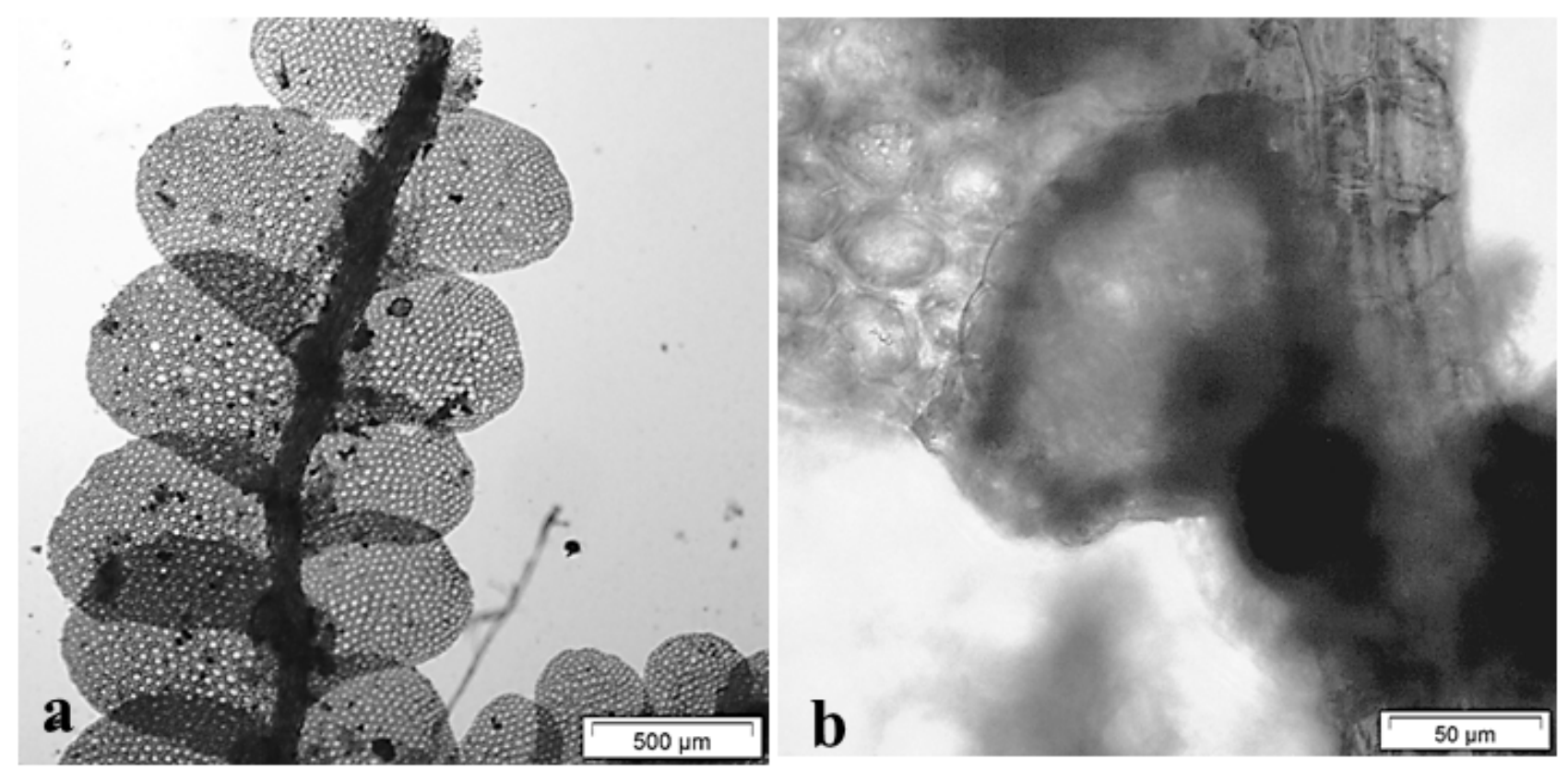

Figure 1. Pycnolejeunea chocoensis M.E.Reiner \& Gradst. a. gametophyte, ventral view. b. leaf lobule (A.M. Sierra 5104, INPA).

4-6 cells wide. The absence of ocelli is also shared with $P$. remotistipula, but the latter species has rectangular leaf lobule and underleaves with rounded lobes apex.

Pycnolejeunea chocoensis was known only from the Chocó region of Colombia and is being referred for the first time to Brazil.

World distribution: Colombia and Brazil.

Distribution in Brazil: Northern.

Habitat: Rainforest, on living trees.

Selected material examined: BRAZIL. AmAzonAs: Manaus, $80 \mathrm{~km}$ ao norte da cidade de Manaus pela BR174, Projeto de Dinâmica Biológica de Fragmentos Florestais (PDBFF), Reserva do km $41,2^{\circ} 30 \mathrm{~S}, 60^{\circ} \mathrm{W}$, 16-XI-2018, A.M. Sierra 5104 (INPA); C.E. Zartman 10232, 10237 (INPA).

2. Pycnolejeunea contigua (Nees) Grolle, J. Hattori Bot. Lab. 45: 179. 1979. $\equiv$ Jungermannia contigua Nees in Martius, Fl. Brasil. enum. plant.1(2): 360. 1833. Type: BRAZIL. Pará: ad corticem arborum, C.F. Martius s.n. (isotype: G00128260!).

Figures $2 \mathrm{a}-\mathrm{d}, 3 \mathrm{a}-\mathrm{d}$

= Pycnolejeunea bancana Steph., Hedwigia 35: 124. 1896. Type: Indonesia: Insula Banca, J.E. Teysmann s.n. (holotype: G 00281813!).

= Pycnolejeunea papulosa Steph., Hedwigia 35: 125. 1896. Type: BRAZIL. Pará: Caripi, R. Spruce s.n. (holotype: G00128263!).

= Pycnolejeunea densiuscula Spruce ex Steph., Sp. Hepat. 5: 613. 1914. Type: BRAZIL. Pará: In cortice ad flum. Amazonum, Obidus, Dec. 1849, R. Spruce s.n. (holotype: G 00128259 ! isotype: G 00128258 !).
= Pycnolejeunea ocellata Steph., Sp. Hepat. 5: 614. 1914.

Type: CUBA. C. Wright s.n. (holotype: G 00128226!).

Description and illustration: He (1999), Gradstein \& IlkiuBorges (2009).

Plants 0.9-1.0 mm wide. Stem 100-110 $\mu \mathrm{m}$ in diameter, in cross section with 9 epidermal cells and 10 medullary cells, thick-walled; ventral merophyte two cells wide. Leaves imbricate, wide-spreading; leaf lobe ovate, 350-550 $\mu \mathrm{m}$ long $\times 300-450 \mu \mathrm{m}$ wide, dorsal margin arched, entire to slightly crenulate, ventral margin arched, entire, apex rounded; leaf cells hexagonal, 12-25 $\mu \mathrm{m} \times 11-18 \mu \mathrm{m}$, weakly papillose (unipapillose), with low papillae, trigones large to small, triangular, intermediate thickenings absent; ocelli (0-)4-7 basal, 16-30 $\mu \mathrm{m} \times 11-15 \mu \mathrm{m}$; oil bodies 1-2 large of the Calypogeia-type; leaf lobule inflated, small, 100-166 $\mu \mathrm{m}$ long $\times 80-120 \mu \mathrm{m}$ wide, ovate, free margin slightly involute, formed by 4-7 elongated cells, apical tooth short, keel arched. Underleaves imbricate, rare contiguous, ovate to suborbicular, $180-300 \mu \mathrm{m}$ long $\times 300-400 \mu \mathrm{m}$ wide, wider than long, $3-5 \times$ stem width, bifid to $1 / 2$ of its length, sinus $\mathrm{V}$-shaped, bases rounded to cuneate, insertion line arched. Autoicous. Androecia on short branches, 2-4 pairs of bracts, bracteole restricted at the base of the branch. Gynoecia on short branch, bract lobe obovate, margins entire, smooth, bracteole bifid; perianths obovate, 5-keeled, keels smooth, beak short. Vegetative reproduction by caducous leaves and regenerants.

Pycnolejeunea contigua can be recognized by ventral merophyte of two cells wide, free margin of the leaf lobule formed by 4-7 cells, imbricate underleaves, bifid to $1 / 2$ of its length, ovate to orbicular, wider than long, $3-5 \times$ stem 

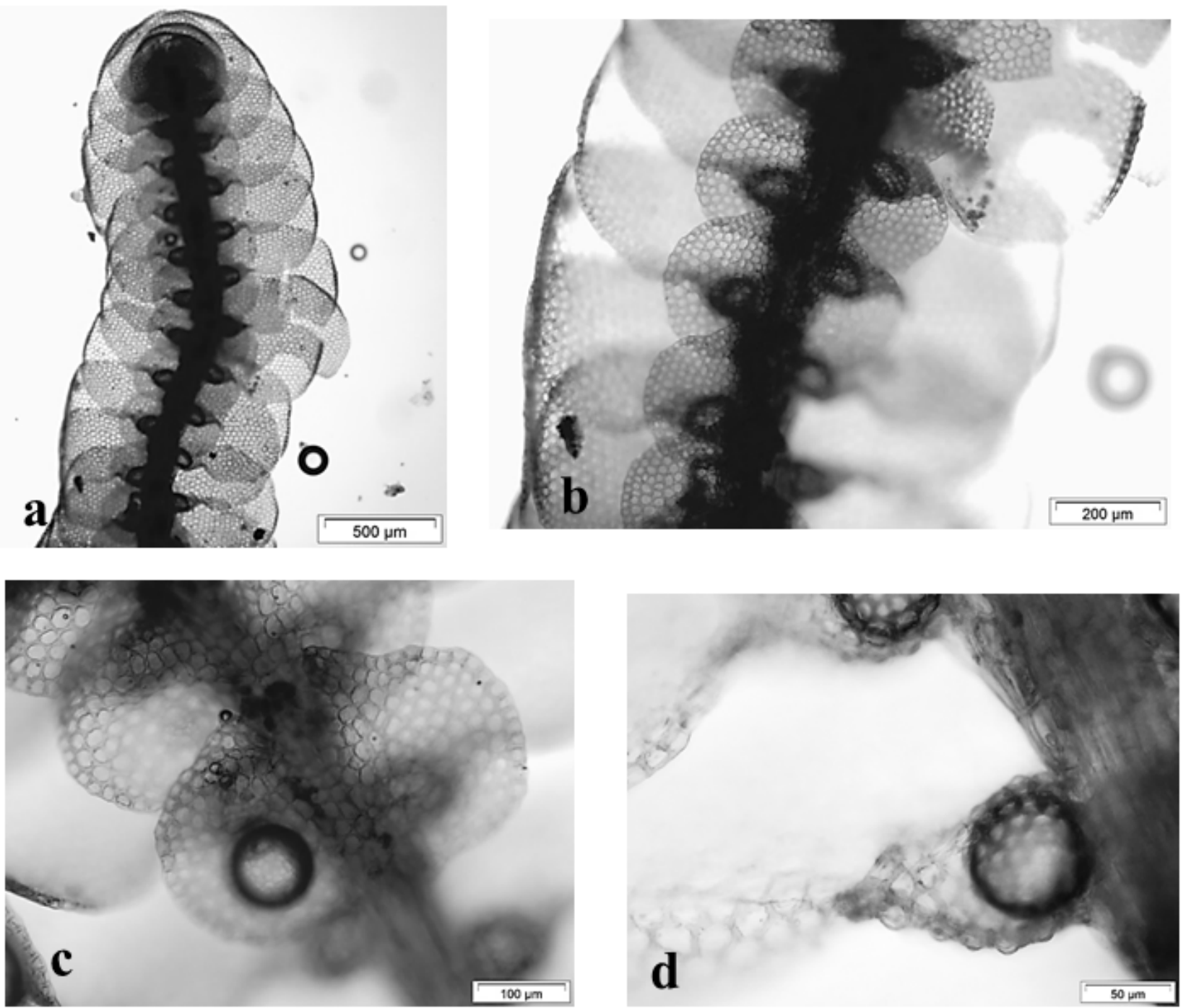

Figure 2. Pycnolejeunea contigua (Nees) Grolle. a. gametophyte, ventral view. b. part of gametophyte with underleaves. c. underleaves. d. leaf lobule (C.F. Martius s.n., G00128260).

width, weakly papillose leaf cells with low papillae; (0-)4-7 basal ocelli; and autoicous.

World distribution: Pantropical.

Distribution in Brazil: Northern, Northeastern, Southeastern, Southern (Yano 2008, 2012, Bastos \& Vilas Bôas-Bastos 2019).

Habitat: Rainforest, seasonal forest, restinga, cerrado, on living trees, leaves and rotten logs.

Selected material examined: BRAZIL. AmazonAs: Barcelos, Serra do Aracá, Serrinha, alto do Rio Aracá, $0^{\circ} 24^{\prime} 53^{\prime} \mathrm{N}, 63^{\circ} 23^{\prime} 08^{\prime} \mathrm{W}$, elev. $300 \mathrm{~m}, 18$-VIII-2014, C.E. Zartman 9638 (ALCB); Manaus, between Manaus and São Gabriel: above and below junction of Rio Branco, E of Carvoeiro, at Paraná da Floresta and Praia do Gavião, igapó at high water, $01^{\circ} 25^{\prime} \mathrm{S}, 61^{\circ} 05-20^{\prime} \mathrm{W}, 26-\mathrm{IV}-1979$, R.M. Schuster 79-2-24 (F); just E of Santa Isabel, igapó and small islands, $00^{\circ} 30^{\prime} \mathrm{S}, 65^{\circ} 00^{\prime} \mathrm{W}, 1-\mathrm{VII}-1979$, R.M. Schuster 79-8-195a (F); Ilha da Costa do Arirarrá, forest just above high water line, $00^{\circ} 25^{\prime} \mathrm{S}, 63^{\circ} 45^{\prime} \mathrm{W}, 28$-VI-1979, R.M. Schuster 79-5-137 (F); São Luíz, $00^{\circ} 10^{\prime} \mathrm{S}, 63^{\circ} 00^{\prime} \mathrm{W}$, 28-VI-1979, R.M. Schuster 79-4-109 (F); Temendui Lagoon, campina, $00^{\circ} 35^{\prime}$ 'S, $64^{\circ} 40^{\prime} \mathrm{W}, 30$-VI-1979, R.M. Schuster 79-7-183 (F); Serra Negra, 31-III-1971, G.T. Prance et al. 11717 (INPA); São Gabriel da Cachoeira, Rio Negro, 20 km descendo da cidade de São Gabriel da Cachoeira, Ilha Corieiari, $00^{\circ} 11$ '53.5” S, 6700'20.5” W, 17-XII-2017, A.M. Sierra 4734 (INPA). Bahia: Entre Rios, Subaúma, $11^{\circ} 56^{\prime} \mathrm{S}, 38^{\circ} 05^{\prime} \mathrm{W}, 25-\mathrm{II}-2000$, C. Bastos 2106 (ALCB); Eunápolis, Estação Veracel, $16^{\circ} 22^{\prime} \mathrm{S}, 39^{\circ} 10^{\prime} \mathrm{W}$, elev. $70-100$ $\mathrm{m}$, estrada da divisa com Imbiruçu, 10-III-2001, C. Bastos 2728 (ALCB); Igrapiúna, Reserva Ecológica da Michelin, $13^{\circ} 50^{\prime} \mathrm{S}, 39^{\circ} 10^{\circ} \mathrm{W}$, elev. 92-383 m, Fragmento Luís Inácio, 3-IV-2010, H.C. Oliveira 1665 (ALCB); Mata de São João, Reserva Ecológica da Sapiranga, Trilha das Bromélias, 21-IV-2005, M. Santos 4 (ALCB); Santa Cruz Cabrália, Fragmento Cara-branca, 9-III-2001, C. Bastos 2740 (ALCB); 

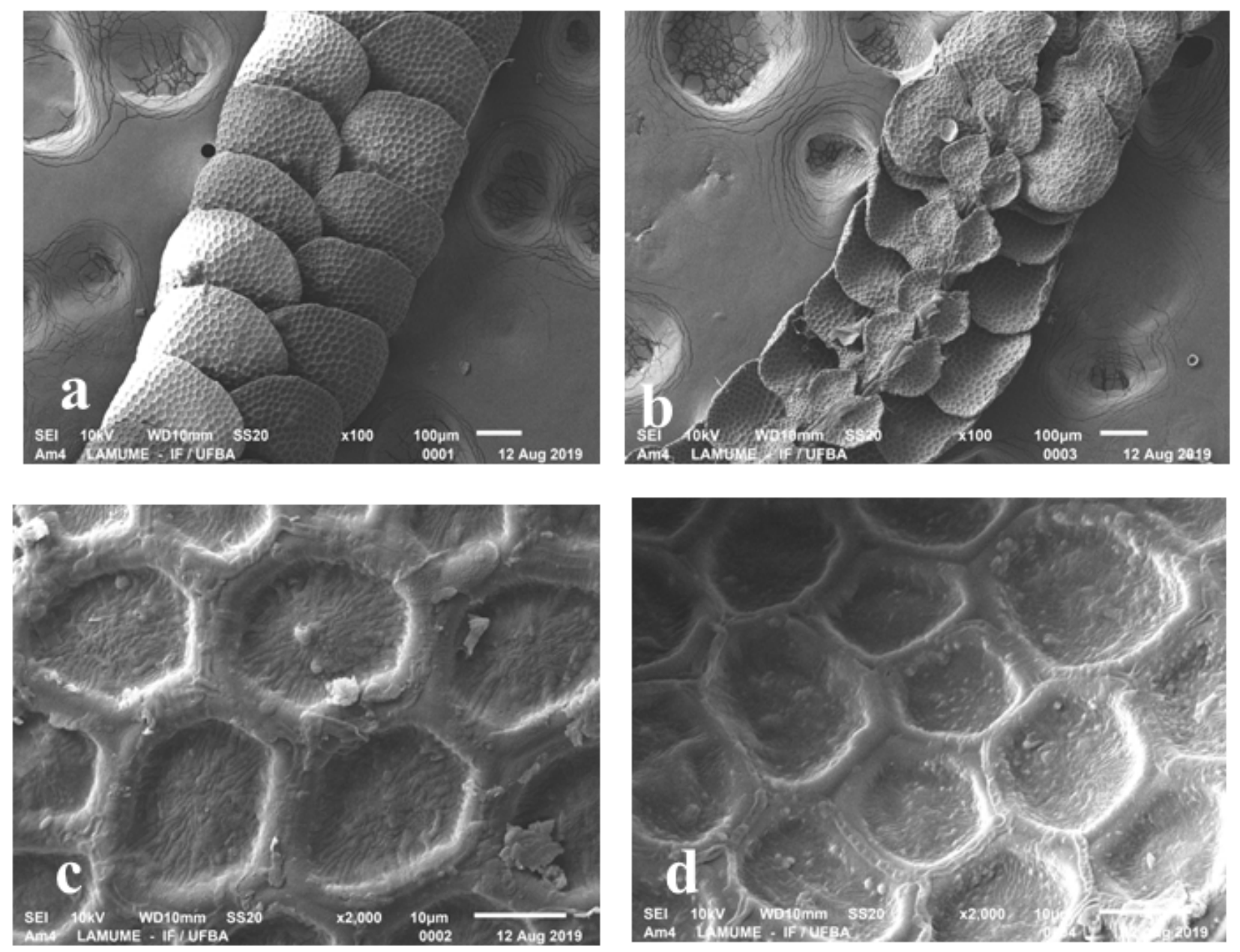

Figure 3. Pycnolejeunea contigua (Nees) Grolle. (SEM). a. gametophyte, dorsal view. b. gametophyte, ventral view. c. dorsal cell surface. d. ventral cell surface (C. Bastos 6449, ALCB).

Boa Nova, Parque Nacional de Boa Nova, Três Cachoeiras, $14^{\circ} 25^{\prime} 41^{\prime \prime}$ 'S, 4007'14'W, elev. 750 m, 7-XI-2018, C. Bastos 6449 (ALCB). Pará: Capitão Poço, estrada da Fazenda São Marcos, $1^{\circ} 46^{\prime} 00^{\prime}$ 'S, 47 $04^{\prime} 00^{\prime \prime} \mathrm{W}$, elev, 40 m, 1-VIII-2005, A.C. Tavares \& M. Rosa 785 (RB); Serra dos Carajás, Serra Sul, S11-A, 6²0'56.9”'S, 50²6'58.1'W, elev. 714 m, 28IV-2015, A.L. Ilkiu-Borges et al. 3436 (MG).

\section{Pycnolejeunea decurviloba Steph., Hedwigia 35: 125.} 1896.

$\equiv$ Cheilolejeunea decurviloba (Steph.) X.L. He, Ann. Bot. Fennici 33: 51. 1996. Type: TRINIDAD: "Tucuche Insula Trinidad Antill", 4-XII-1847, H. Crüger 5 (lectotype: G, not seen).

Description and illustration: He (1996, as Cheilolejeunea decurviloba).

Plants 1.7-1.8 mm wide. Stem $180 \mu \mathrm{m}$ in diameter, in cross section with 13 epidermal cells and 17-19 medullary cells, thick-walled; ventral merophyte $4-6$ cells wide. Leaves imbricate, spreading; leaf lobe ovate, $0.8-1.3 \mathrm{~mm}$ long $\times$
0.7-0.8 mm wide, convex, dorsal margin slightly arched, entire, ventral margin straight, entire, apex rounded, mostly curved; cells oblong to hexagonal, $23-45 \mu \mathrm{m} \times 15-20 \mu \mathrm{m}$, mammillose, trigones large, intermediate thickenings indistinct; ocelli absent; oil bodies not seen; leaf lobule ovate, $190-230 \mu \mathrm{m}$ long $\times 130-140 \mu \mathrm{m}$ wide, decurved, free margin plane, arched, composed by 5-6 cells, tooth acute, falcate, apical margin strongly curved, keel concave. Underleaves imbricate, reniform, 0.4-0.6 mm long $\times$ 0.9-1.3 $\mathrm{mm}$ wide, $5-6 \times$ stem width, bifid to $1 / 4-1 / 5$ of its length, bases cuneate, insertion line deeply arched. Androecia not seen. Gynoecia on main stem, 1-2 pycnolejeuneoid innovation, bracts lobe obovate, margins entire, lobule obovate, apex acute, bracteole widely ovate, short-bifid, perianth not seen.

Pycnolejeunea decurviloba is characterized by ventral merophyte with 4-6 cells wide, imbricate leaves; ovate leaf lobe, ocelli lacking, lobule decurved; plane free margin, arched, composed by 5-6 cells; falcate apical tooth; and reniform, imbricate underleaves. $P$. decurviloba shares 
with $P$. chocoensis the decurved leaf lobule and absence of ocelli, but in the latter species the underleaves are ovate and distant, and the ventral merophyte has two cells wide.

World distribution: Tropical America.

Distribution in Brazil: Northern.

Habitat: Rainforest, on living trees.

Selected material examined: BRAZIL. RoRAIMA: Vicinity of Aquaris, $4^{\circ} 6^{\prime} \mathrm{N}, 64^{\circ} 25^{\prime} \mathrm{W}$, elev. $800 \mathrm{~m}$, low caatinga forest, growing on living tree, 28-VII-1974, G.T Prance et al. 21493 (INPA).

4. Pycnolejeunea densistipula (Lehm. \& Lindenb.) Steph., Sp. Hepat. 5: 602. 1914.

三Lejeunea densistipula Lehm. \& Lindenb., Nov. Stirp. Pug. 7: 20. 1838. Type: PERU: "Ad corticem officinalem quandam, quae agnosci non potui, reprimus", s.d., s.leg., (isotype: $\mathrm{W}$, not seen).

Description and illustration: He (1999).

Plants 1.3-1.7 mm wide. Stem $250 \mu \mathrm{m}$ in diameter, in cross section with 14-26 epidermal cells and 30-57 medullary cells, thick-walled; ventral merophyte 4-8 cells wide. Leaves imbricate, spreading; leaf lobe ovate-orbicular, $0.7-1.0 \mathrm{~mm}$ long $\times 0.7-0.8 \mathrm{~mm}$ wide, dorsal margin arched, entire, ventral margin straight, entire, apex rounded, plane to curved; leaf cells hexagonal, 23-30 $\mu \mathrm{m} \times 20-23 \mu \mathrm{m}$, mammillose, trigones small to large, intermediate thickenings occasional; ocelli basal to suprabasal, $45-63 \mu \mathrm{m} \times 35-38 \mu \mathrm{m}$; oil bodies not seen; leaf lobule ovate, small, $110-150 \mu \mathrm{m}$ long $\times 70-80 \mu \mathrm{m}$ wide, free margin slightly involute to flat, formed by 4-5(-7) cells, tooth short, keel slightly arched, crenulate; reduced lobules occasional. Underleaves large, imbricate, reniform to ovate to suborbicular, 360-500 $\mu \mathrm{m}$ long $\times 430-800 \mu \mathrm{m}$ wide, bifid to $1 / 5$ of its length, sinus acute, margin revolute or plane, bases straight to rounded, insertion line curved. Androecia on short branches, 2-3 pairs of bracts, bracteoles restricted to the base of the branch. Gynoecia not seen. Vegetative reproduction by caducous leaves.

Pycnolejeunea densistipula is characterized by ventral merophytes of 4-8 cells wide, basal to suprabasal ocelli, small leaf lobule (ca. 110-150 $\mu \mathrm{m}$ long), involute, free margin formed by 4-5(-7) cells, short apical tooth, imbricate underleaves, reniform to ovate to suborbicular, bifid to $1 / 5$ of its length, revolute to plane margin.

World distribution: Widespread in tropical America (He 1999).

Distribution in Brazil: Northern, Northeastern, Southeastern (Yano 2008, Bastos \& Vilas Bôas-Bastos 2019).

Habitat: Rainforest and restinga, on living trees and rotten logs.

Selected material examined: BRAZIL. BAHIA: Igrapiúna, Reserva Ecológica da Michelin, $13^{\circ} 48^{\prime} \mathrm{S}, 39^{\circ} 10^{\prime} \mathrm{W}$, elev. 90-380 m, Fragmento Pacangê, 8-II-2008, S.B. Vilas Bôas-
Bastos 2349, 2350; C. Bastos 4992 (ALCB); Wenceslau Guimarães, Estação Ecológica de Wenceslau Guimarães, Trilha Serra Grande, $13^{\circ} 35^{\prime} 43^{\prime \prime S}, 39^{\circ} 43^{\prime} 13^{\prime \prime} \mathrm{W}$, elev. $583 \mathrm{~m}$, 28-IX-2017, S.B. Vilas Bôas-Bastos 3348 (ALCB).

5. Pycnolejeunea gradsteinii Ilk.-Borg., Boletim do Instituto de Botânica 21: 1. 2011.

Type: BRAZIL. Pará: Melgaço, Caxiunã National Forest, Ferreira Penna Research Station, Tijucaquara creek, terra firme forest, on living trunk, 9-I-1999, A.L. Ilkiu-Borges \& C. Zartman 1294 (holotype: MG!).

Description and illustration: Ilkiu-Borges (2011).

Plants 400-600 $\mu \mathrm{m}$ wide. Stem $50 \mu \mathrm{m}$ in diameter, in cross section with 7-9 epidermal cells and 7-9 medullary cells, thick-walled; ventral merophyte two cells wide. Leaves imbricate, spreading; leaf lobe suborbicular to ovate, $280-400$ $\mu \mathrm{m}$ long $\times 230-300 \mu \mathrm{m}$ wide, dorsal margin arched, entire, ventral margin straight, entire, apex rounded to acute; cells hexagonal, $25-40 \mu \mathrm{m} \times 18-20 \mu \mathrm{m}$, unipapillose, trigones small to indistinct, intermediate thickenings absent; ocelli 1-2 basal, $18-20 \mu \mathrm{m} \times 25-40 \mu \mathrm{m}$; oil bodies not seen; leaf lobule conic, $120-130 \mu \mathrm{m}$ long $\times 80-100 \mu \mathrm{m}$ wide, free margin plane to slightly involute, formed by 7-9 elongated cells, tooth short, acute, slightly falcate, apical margin deeply curved, keel straight to slightly arched. Underleaves ovate to suborbicular, $130-160 \mu \mathrm{m}$ long $\times 130-140 \mu \mathrm{m}$ wide, bifid to $1 / 2$ of its length, sinus acute, bases cuneate, insertion line straight to curved. Androecia and gynoecia not seen.

Pycnolejeunea gradsteinii is very similar to Pycnolejeunea porrectilobula, especially in the conical shape of the lobe, with a narrow U-shaped apex. However, differs from this latter species in the following characters: (1) ovate leaf lobe (oblong-ovate in P. porrectilobula); (2) small to indistinct trigones (larger trigones in P. porrectilobula); (3) distant underleaves (imbricate to contiguous in P. porrectilobula); (4) unipapillose leaf cells (pluripapillose leaf cells in $P$. porrectilobula).

World distribution: Brazil.

Distribution in Brazil: Northern.

Habitat: Rainforest, on living trees.

6. Pycnolejeunea macroloba (Nees \& Mont.) Schiffn., in Engler \& Prantl, Nat. Pflanzenfam. 1(3): 124. 1893. 三 Lejeunea macroloba Nees \& Mont., Ann. Sci. Nat. Bot., ser. 2, 19: 260. 1843. Type: GUYANA. "Ad truncus arborum in sinnamariensi necropoli”. Rara. s.d., Lepr. Coll. $n .273$ (isotype: JE, not seen).

= Pycnolejeunea callosa (Lindenb.) Steph., Sp. Hepat. 5: 609. 1914. Lejeunea callosa Lindenb., Type: BRAZIL. “Ad flumen Amazonum”, J.F. Sehlmeyer 467 (isotype: G 00128809!).

Description and illustration: He (1999), Gradstein \& IlkiuBorges (2009). 
Plants $1.3-1.7 \mathrm{~mm}$ wide. Stem $150 \mu \mathrm{m}$ in diameter, in cross section with 10 epidermal cells and 18 medullary cells; ventral merophyte two cells wide. Leaves imbricates, spreading; leaf lobe oblong-orbicular, 600-900 $\mu \mathrm{m}$ long $\times$ 400-600 $\mu \mathrm{m}$ wide, dorsal margin arched, entire, ventral margin slightly arched, apex rounded; cells oblong, 24$38 \mu \mathrm{m} \times 16-24 \mu \mathrm{m}$, mamillose, trigones large, triangular, intermediate thickenings absent; ocelli 2-4(-5) basal to suprabasal, $50-65 \mu \mathrm{m} \times 28-33 \mu \mathrm{m}$; oil bodies nor seen; leaf lobule large, $312-480 \mu \mathrm{m}$ long $\times 136-184 \mu \mathrm{m}$ wide, inflated, rectangular, free margin plane to slightly involute, formed by 9-20 elongated cells, tooth short, keel straight. Underleaves large, 300-400 $\mu \mathrm{m}$ long $\times 300-600 \mu \mathrm{m}$ wide, suborbicular, wider than long, imbricates to contiguous, bifid to $1 / 2$ of its length, sinus acute, bases rounded, insertion line arched. Androecia not found. Gynoecia on main stem, innovation not seen, bracts lobe obovate, margins entire, apex rounded, lobule large, rectangular, bracteole widely ovate, perianths not seen.

The main characters of Pycnolejeunea macroloba are the large, rectangular leaf lobule with free margin composed by $9-20$ cells, and large underleaves (300-600 $\mu \mathrm{m}$ wide), mostly imbricate underleaves.

World distribution: Widespread in tropical America (He 1999).

Distribution in Brazil: Northern, Northeastern, Southeastern (Yano 2008, 2012, Bastos \& Vilas BôasBastos 2019).

Habitat: Rainforest, on living trees.

Selected material examined: BRAZIL. AmAZONAS: Manaus, Caracaraí Road, km 45, 5-IV-1971, G.T. Prance et al. 11374, 11386 (INPA); SESC, estrada dos Franceses, km
5, balneário, 21-VII-1974, O. Yano 257 (INPA). BAHIA: Eunápolis, Estação Veracel, $16^{\circ} 22^{\prime} \mathrm{S}, 39^{\circ} 10^{\prime} \mathrm{W}$, elev. 70100 m, Estrada do Meio, 11-VI-1999, S.B. Vilas BôasBastos \& C. Bastos 748 (ALCB); Igrapiúna, Reserva Ecológica da Michelin, $13^{\circ} 50^{\prime} \mathrm{S}, 39^{\circ} 10^{\prime} \mathrm{W}$, elev. 90-383 m, Fragmento Luís Inácio, 4-VIII-2010, H.C. Oliveira 1861 (ALCB); Una, Reserva Biológica de Una, Trilha do Príncipe, 15¹0'55"S, 3904'27’'W, elev. 97 m, C. Bastos 6595 (ALCB). PARÁ: Capitão Poço, Fazenda São Marcos, $1^{\circ} 46^{\prime} 00^{\prime}$ 'S, 4704'00”'W, elev. 40 m, 15-IX-2006, A.C. Tavares \& D. Nascimento 1011 (RB).

7. Pycnolejeunea papillosa X.-L. He, Acta Bot. Fenn. 163: 55, f. 17: a-p. 1999. Type: VENEZUELA. 17-X-1987, R.E. Halling 5562 (holotype: NY, not seen).

Description and illustration: He (1999).

Figure $4 \mathrm{a}, \mathrm{b}$

Plants $600-700 \mu \mathrm{m}$ wide. Stem $100 \mu \mathrm{m}$ in diameter, in cross section with 7 epidermal cells and 9-10 medullary cells, thick-walled; ventral merophyte two cells wide. Leaves imbricate, spreading to slightly falcate; leaf lobe orbicular-ovate, $350-460 \mu \mathrm{m}$ long $\times 320-430 \mu \mathrm{m}$ wide, convex, dorsal margin arched, crenulate, ventral margin slightly arched, crenulate, apex rounded, plane to curved; leaf cells hexagonal, $23-30 \mu \mathrm{m} \times 18-25 \mu \mathrm{m}$, strongly papillose (unipapillose), with high papillae, trigone small to indistinct, intermediate thickenings absent; ocelli 1-2 basal, 43-50 $\mu \mathrm{m}$ $\times 28-30 \mu \mathrm{m}$; oil bodies not seen; leaf lobule ovate, inflated, $150-200 \mu \mathrm{m}$ long $\times 90-110 \mu \mathrm{m}$ wide, free margin involute, formed by 5-6 elongated cells, apical tooth short, keel arched, lobule cells strongly unipapillose, cells with huge papillae,
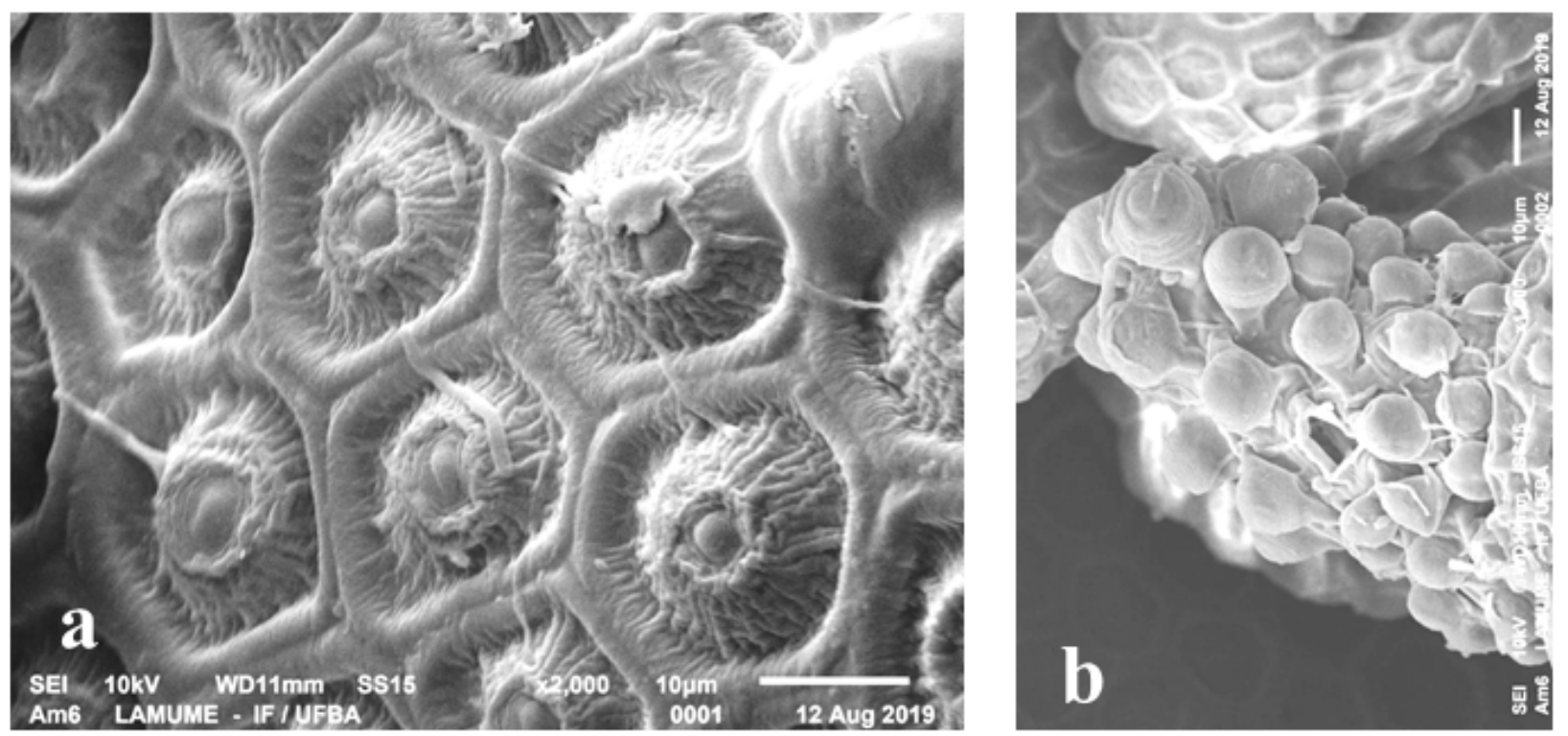

Figure 4. Pycnolejeunea papillosa X.-L. He (SEM). a. dorsal cell surface showing papillose cells. b. surface of the leaf lobule keel showing the strongly papillose cells (C.E. Zartman 9646, ALCB). 
$18-25 \mu \mathrm{m}$ high $\times 15-20 \mu \mathrm{m}$ at base. Underleaves imbricate, orbicular to reniform, $160-180 \mu \mathrm{m}$ long $\times 240-310 \mu \mathrm{m}$ wide, bifid to $1 / 3$ of its length, sinus acute to U-shaped, bases cuneate, insertion line curved. Androecia not seen. Gynoecia on main stem, with one innovation, bracts lobe obovate, margins crenulate, lobule obovate, bracteole ovate, bifid, perianth not seen.

Pycnolejeunea papillosa is characterized by leaf lobe and lobule cells with huge, rounded papillae (see figure 4), free margin of leaf lobule composed by 5-6 cells, and imbricate, orbicular to reniform underleaves. According to He (1999), P. papillosa can be confused with P. contigua, but $P$. papillosa is a very different plant due to leaf cells with a huge, rounded papillae, strongly convex leaves and leaf lobe margins crenulate.

World distribution: Brazil and Venezuela (He 1999).

Distribution in Brazil: Northern, Northeastern, Southeastern (Yano 2008, 2012, Bastos \& Vilas BôasBastos 2019).

Habitat: Rain forest, on living trees.

Selected material examined: BRAZIL. AmAzonas: Barcelos, Serra do Aracá, 0 ${ }^{\circ} 52^{\prime} 04^{\prime \prime} \mathrm{N}, 63^{\circ} 19^{\prime} 56^{\prime}$ 'W, elev. $1300 \mathrm{~m}, 18$ VIII-2014, C.E. Zartman 9646 (INPA, ALCB); São Gabriel da Cachoeira, Reserva Morro dos Seis Lagos, 29-VIII-2011, C.E. Zartman 8657 (INPA, ALCB). BAHIA: Wenceslau Guimarães, Estação Ecológica de Wenceslau Guimarães, Trilha Serra Grande, $13^{\circ} 35^{\prime} 43^{\prime \prime S}, 39^{\circ} 43^{\prime} 13^{\prime \prime} \mathrm{W}$, elev. 583 m, 28-IX-2017, C. Bastos 5987p.p. (ALCB).

8. Pycnolejeunea porrectilobula C.J. Bastos \& O. Yano, Nova Hedwigia 74(3-4): 440. 2002.

Type: BRAZIL. Bahia: Eunápolis, Estação Veracruz, 10-IX1999, C. Bastos \& S.B. Vilas Bôas-Bastos 1824 (holotype: ALCB!).

Figure $5 \mathrm{a}, \mathrm{b}$

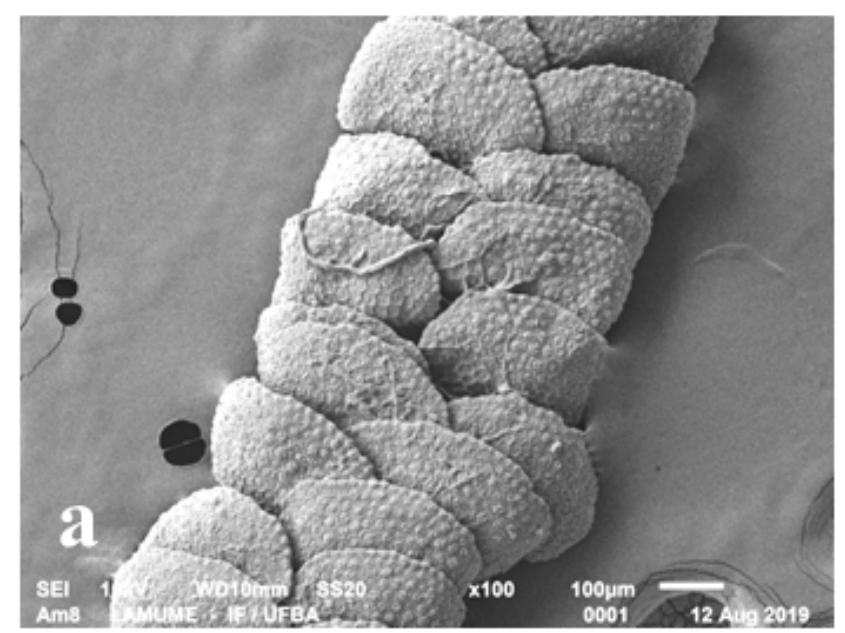

Description and illustration: Bastos \& Yano (2002).

Plants 0.8-1.2 mm wide; flagelliform branches with reduced leaves occasional. Stem $110 \mu \mathrm{m}$ in diameter, in cross section with 7-8 epidermal cells and 8-10 medullary cells, thick-walled; ventral merophyte two cells wide. Leaves imbricate, spreading to falcate; leaf lobe convex, oblongovate, falcate, $496-512 \mu \mathrm{m}$ long $\times 335-400 \mu \mathrm{m}$ wide, dorsal margin arched, crenulate, ventral margin straight to slightly arched, crenulate, apex acute to obtuse to rounded; leaf cells oblong, 20-36 $\mu \mathrm{m} \times 18-26 \mu \mathrm{m}$, strongly pluripapillose, trigones large, intermediate thickenings 1-2 per cell; ocelli 1-4 basal, 53-65 $\mu \mathrm{m} \times 30-33 \mu \mathrm{m}$; oil bodies 2-3 per cell, coarsely granular; leaf lobule conic, large, 176-264 $\mu \mathrm{m}$ long $\times 88-112 \mu \mathrm{m}$ wide, free margin slightly involute to plane, with 6-9 rectangular cells, apical tooth acute to obtuse, apical margin U-shaped, sinus narrow, keel straight, strongly crenulate. Underleaves large, 220-260 $\mu \mathrm{m}$ long $\times 240$ $360 \mu \mathrm{m}$ wide, orbicular to widely ovate to reniform, wider than long, imbricate to contiguous, bifid to $1 / 2-1 / 3$ of its length, sinus acute, bases rounded, insertion line slightly arched. Androecia not seen. Gynoecia on main stem, with one pycnolejeuneoid innovation, bracts spreading, lobe obovate-oblong, margins crenulate, apex rounded, curved, lobule oblong, apex acute to emarginated, bracteole widely ovate, bifid to $1 / 4-1 / 5$ of its length, sinus acute; perianths obovate, 5-keeled, keels crenulate. Vegetative multiplication by gemmae and fragmentation.

Pycnolejeunea porrectilobula is characterized by convex, falcate leaf lobe, large, conic leaf lobule, free margin with 6-9 rectangular cells, imbricate to contiguous underleaves, wider than long, widely ovate to orbicular to reniform, 1-4 basal ocelli, strongly pluripapillose leaf cells (figure 4). Due to it lobule shape, P. porrectilobula resembles $P$. gradsteinii in the lobule shape but differs from the latter species in the pluripapillose leaf cells (unipapillose in P. gradsteinii), convex and falcate leaf lobe (plane, not falcate leaf lobe

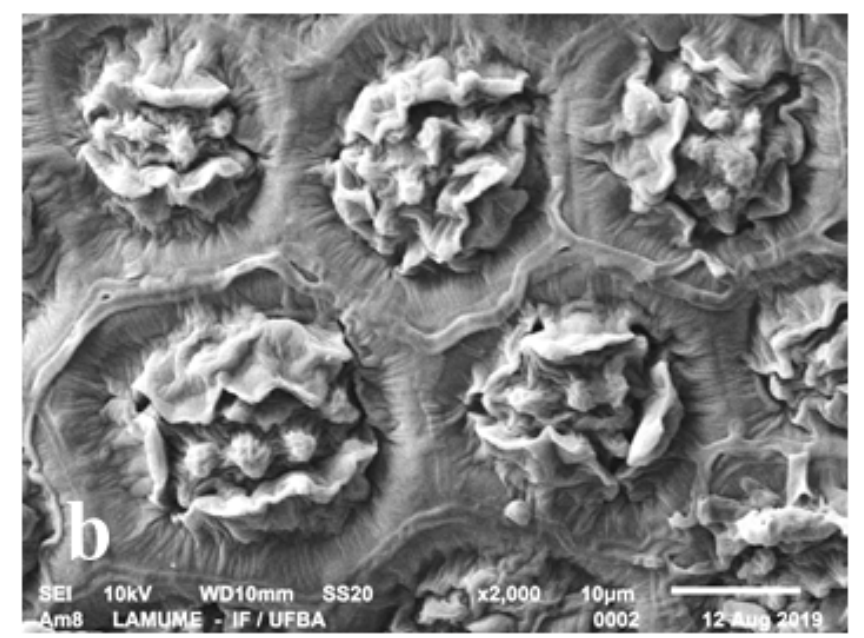

Figure 5. Pycnolejeunea porrectilobula C.J. Bastos \& O. Yano (SEM). a. gametophyte, dorsal view. b. dorsal cell surface showing the strongly pluripapillose cells (from holotype, C. Bastos et al. 1824, ALCB). 
in P. gradsteinii), and imbricate, widely ovate to reniform underleaves (distant, ovate underleaves in $P$. gradsteinii).

In the original description of Pycnolejeunea porrectilobula the presence of only one ocellus is mentioned, but in the other materials examined, 2-4 ocelli were found. The presence of pluripapillose leaf cells was also not mentioned in the original description of $P$. porrectilobula. To date, P. porrectilobula has only been reported for the State of Bahia.

World distribution: Brazil.

Distribution in Brazil: Northeastern (Bahia).

Habitat: Rainforest, on living trees.

Further selected material examined: BRAZIL. BAHIA: Eunápolis, Estação Veracruz, $16^{\circ} 22^{\prime} \mathrm{S}, 39^{\circ} 10^{\prime} \mathrm{W}$, elev. 70-100 m, Picada do Encontro dos Rios, 23-III-2000, S.B. Vilas Bôas-Bastos \& C. Bastos 1547 (ALCB); Una, Reserva Biológica de Una, Trilha do Príncipe, $15^{\circ} 10^{\prime} 55^{\prime}$ 'S, 3904'27'W, elev. 97 m, 12-II-2019, S.B. Vilas Bôas-Bastos 3718 (ALCB).

9. Pycnolejeunea remotistipula C.J. Bastos \& C.E. Zartman, Neodiversity 10: 2. 2017.

Type: BRAZIL. Amazonas: Rio Negro, between Manaus and São Gabriel, along BR 307, from São Gabriel, just N of igarapé Iá-Mirim, near Jerusalém, primary forest, $00^{\circ} 20^{\prime} \mathrm{N}, 66^{\circ} 35^{\prime} \mathrm{W}$, 17-VII-1979, R.M. Schuster 79-18-935 (holotype: F!).

Description and illustration: Bastos \& Zartman (2017).

Plants 1.4-1.5 mm wide, Stem $100 \mu \mathrm{m}$ in diameter, in cross section with 7 epidermal cells and 8-9 medullary cells; ventral merophyte two cells wide. Leaves imbricate, spreading; leaf lobe ovate-oblong, flat, 500-700 $\mu \mathrm{m}$ long $\times 340-510 \mu \mathrm{m}$ wide, dorsal margin slightly arched, entire, ventral margin slightly arched to straight, entire, apex rounded; median cells hexagonal, 15-28 $\mu \mathrm{m} \times 13-18 \mu \mathrm{m}$, slightly mammillose to plane, trigones small, intermediate thickenings 1-2 per cell, basal cells 40-45 $\mu \mathrm{m} \times 23-25 \mu \mathrm{m}$, forming a weak vitta; ocelli absent; oil bodies not seen; leaf lobule large, rectangular-ovate, 290-360 $\mu \mathrm{m}$ long $\times$ $150-190 \mu \mathrm{m}$ wide, slightly inflated, free margin slightly involute, formed by 15-17 elongated cells, strongly thickwalled, apical tooth short, straight to vertically oriented, keel straight, smooth to weakly crenulate. Underleaves distant, small, $180-220 \mu \mathrm{m}$ long $\times 170-210 \mu \mathrm{m}$ wide, bifid to $1 / 2$ of its length, sinus acute, lobes obtuse, apex rounded, bases cuneate, insertion line curved. Autoicous. Androecia on main stem, 4-5 pairs of bracts, bracteoles at the base of branch. Gynoecia on short branch, innovation not seen, bracts lobe obovate, margins entire, apex rounded, lobule oblong, apex acute, bracteole short-bifid to emarginated; perianths oblong, 5-keeled, keels smooth, beak short.

Pycnolejeunea remotistipula is characterized by plane leaf lobe, absence of ocelli, distant underleaves, bifid to $1 / 2$ of its length, rounded lobes apices, large leaf lobule
(290-360 $\mu \mathrm{m}$ long), rectangular-ovate, free margin formed by 15-17 elongated cells, strongly thickened. In the original description of $P$. remotistipula an error occurred in the citation of the collector number of the holotype and here was corrected: R.M. Schuster 79-18-835 to 79-18-935.

World distribution: Brazil.

Distribution in Brazil: Northern.

Habitat: Rainforest, on living tree or rock.

Selected material examined: BRAZIL. AmAzonAs: highway between Humaitá and Porto Velho, $60 \mathrm{~km}$ of Humaitá, at highway crossing of São João stream, ca. $8^{\circ} \mathrm{S}, 63^{\circ} \mathrm{W}$, 30-IV-1982, A.J. Fife et al. 3982 (INPA); São Gabriel da Cachoeira, $20 \mathrm{~km}$ descendo o Rio Negro de São Gabriel, $00^{\circ} 10^{\prime} 47.5^{\prime \prime}$ S, 6701'25.0” W, 16-XII -2017, Sierra, A.M. 4702, 4703 (INPA).

\section{Acknowledgments}

The authors are grateful to curators of the herbaria F, G, INPA, MG, SP, RB, for the loan of specimens, and to the Universidade Federal da Bahia, for supporting fieldwork; to Laboratório Multiusuário de Microscopia Eletrônica (LAMUME) of the Instituto de Física, Universidade Federal da Bahia, for making the SEM photographs. We thank the Biological Dynamics of Forest Fragments Project (PDBFF), Manaus, Brazil and the Amazon Fertilization Experiment (AFEX), for support during fieldwork.

\section{Literature cited}

Bastos, C.J.P. 2010. The position of the hyaline papilla and the genus concept of Cheilolejeunea (Spruce) Schiffn. (Lejeuneaceae). Revista Brasileira de Botânica 33: 377-378.

Bastos, C.J.P. 2019. Pycnolejeunea. In: Flora do Brasil 2020 em construção. Jardim Botânico do Rio de Janeiro. Available in http://reflora.jbrj.gov.br/reflora/ floradobrasil/FB97627 (access in 9-XII-2019).

Bastos, C.J.P. \& Vilas Bôas-Bastos, S.B. 2019. Catálogo da família Lejeuneaceae (Marchantiophyta) no Estado da Bahia, Brasil. Pesquisas, Botânica 73: 55-111.

Bastos, C.J.P. \& Yano, O. 2002. Pycnolejeunea porrectilobula (Lejeuneaceae), a new species from Brazil. Nova Hedwigia 74: 477-485.

Bastos, C.J.P. \& Zartman, C.E. 2017. A new species of Pycnolejeunea (Marchantiophyta, Lejeuneaceae) from Brazil. Neodiversity 10: 1-6.

Câmara, P.E.A.S. \& Kellogg, E.A. 2010. Morphology and development of leaf papillae in Sematophyllaceae. The Bryologist 113: 22-33.

Gradstein, S.R. \& Ilkiu-Borges, A.L. 2009. Guide to the Plants of Central French Guiana. Part 4. Liverworts and Hornworts. Memoirs of the New York Botanical Garden 76: 1-140. 
He, X.-L. 1996. On the taxonomic significance of lobule characters in the Lejeuneaceae (Hepaticae). Ann. Bot. Fenn. 33: 311-316.

He, X.-L. 1999. A taxonomic monograph of the genus Pycnolejeunea (Lejeuneaceae, Hepaticae). Acta botanica Fennica 163: 1-77.

Ilkiu-Borges, A.L. 2011. On Pycnolejeunea gradsteinii (Lejeuneaceae), a new species from Brazil. Boletim do Instituto de Botânica 21: 1-4.

Mizutani, M. 1961. Revision of Japanese Lejeuneaceae. Journal of the Hattori Botanical Laboratory 24: 115-302.

Reiner-Drehwald, M.E. \& Gradstein, S.R. 2018. A further new species of Lejeuneaceae (Marchantiophyta) from the Chocó of Colombia: Pycnolejeunea chocoensis. Cryptogamie, Bryologie 39: 325-330.

Renner, M.A.M. 2012. Further insight into lobule teeth homology in Lejeuneaceae subf. Lejeuneoideae from Cheilolejeunea oscilla, a new species from Australia. The Bryologist 115: 536-556.

Schäfer-Verwimp, A., Feldberg, K., Dong, S., van Melick, H., Peralta, D.F., Schmidt, A.R., Schneider, H. \& Heinrichs, J. 2014. Towards a monophyletic classification of Lejeuneaceae III: the systematic position of Leiolejeunea. Phytotaxa 170: 187-198.

Söderström, L., Barrie, F.R., Hagborg, A., CrandallStotler, B.J., Gradstein, S.R., Stotler, R.E. \& Von Konrat, M. 2015. Notes on Early Land Plants Today. 73. Genera of Lejeuneaceae established in the period 18841893: Dates of validation and implications. Phytotaxa 220: 143-198.
Söderström, L., Hagborg, A., Von Konrat, M., Bartholomew-Began, S., Bell, D., Briscoe, L., Brown, E., Cargill, D.C., Cooper, E.D., Costa, D.P., CrandallStotler, B.J., Dauphin, G., Engel, J.J., Feldberg, K., Glenny, D., Gradstein, S.R., He, X., Heinrichs, J., Hentschel, J., Ilkiu-Borges, A.L., Katagiri, T., Konstantinova, N.A., Larraín, J., Long, D.G., Nebel, M., Pócs, T., Puche, F., Reiner-Drehwald, M.E., Renner, M.A.M., Sass-Gyarmati, A., SchäferVerwimp, A., Segarra Moragues, J.G., Stotler, R.E., Sukkharak, P., Thiers, B.M., Uribe, J., Váňa, J., Villarreal, J.C., Wigginton, M., Zhang, L. \& Zhu, R.L. 2016. World checklist of hornworts and liverworts. PhytoKeys 59: 1-828.

Yano, O. 2008. Catálogo de hepaticas e antóceros brasileiros: literatura original, basiônimo, localidadetipo e distribuição geográfica. Boletim do Instituto de Botânica 19: 1-110.

Yano, O. 2012. Catálogo das briófitas (antóceros, hepáticas e musgos) do Estado do Espírito Santo, Brasil. Pesquisas, Botânica 63: 55-140.

Ye, W., Gradstein, S.R., Shaw, A.J., Shaw, B., Ho, B-C., Schäfer-Verwimp, A., Pócs, T., Heinrichs, J. \& Zhu, R.L. 2015. Phylogeny and classification of Lejeuneaceae subtribe Cheilolejeuneinae (Marchantiophyta) based on nuclear and plastid molecular markers. Cryptogamie, Bryologie 36: 313-333.

Zartman, C.E. \& Ilkiu-Borges, A.L. 2007. Guide to the epiphyllous bryophytes of Central Amazonia. Instituto de Pesquisas da Amazônia, Manaus. 